\title{
Light Field Acquisition Method Based on Depth Sampling
}

\author{
Fuzhou Duan, ${ }^{1,2}$ Ying Zuo, ${ }^{1,2 *}$ Hongliang Guan, ${ }^{1,2,3^{* *}}$ and Tian Guo ${ }^{1,2}$ \\ ${ }^{1}$ Engineering Research Center of Spatial Information Technology, MOE, Capital Normal University, \\ 105 West Third Ring North Road, Haidian District, Beijing 100048, China \\ ${ }^{2}$ Key Lab of 3D Information Acquisition and Application, MOE, Capital Normal University, \\ 105 West Third Ring North Road, Haidian District, Beijing 100048, China \\ ${ }^{3}$ Beijing Imaging Technology Innovation Center, Capital Normal University, \\ 105 West Third Ring North Road, Haidian District, Beijing 100048, China
}

(Received July 8, 2021; accepted September 1, 2021; online published September 14, 2021)

Keywords: light field imaging, depth sampling, light field acquisition, clarity evaluation

Light field imaging technology is a new imaging method for recording light propagation. It records light mainly through two devices: a camera array and an all-optical camera. The collected light field data can be processed in different ways such as post hoc on-demand imaging and depth estimation. This technology has high application value in the fields of depth extraction, 3D modeling, and virtual reality, and is one of the hot spots of imaging research. However, existing light field acquisition methods are based on angular sampling, which requires special hardware. The angular and spatial resolutions of the light field collected by these methods are mutually constrained. Accordingly, we propose a new acquisition method for the depth sampling of the light field and compare the clarity of light field subaperture images acquired by this method with that of the images acquired by the traditional angular sampling method. The results of the study show that the spatial resolution of the light field images acquired with depth sampling reaches the sensor's pixel dimensions without special hardware, and their sharpness is very close to that of light field images acquired by the angular sampling method. This study provides a more concise approach to the acquisition of light field images in computational imaging techniques.

\section{Introduction}

Light field imaging is a technique that first acquires an image and then recomputes it using algorithms. $^{(1,2)}$ The imaging process includes two parts: the acquisition of the light field and the processing of the light field data. Light field imaging technology captures the 4D parameter values of light fields and subaperture images from multiple angles in a single exposure, which makes it interesting for applications in depth extraction, 3D modeling, and virtual reality. The results of light field acquisition directly determine whether this technology will have wide application, and much research has been conducted on how to acquire a light field. Adelson and Bergen proposed a 7D all-optical function $P(x, y, z, \theta, \varphi, \lambda, t)$ to characterize the geometric distribution of light in space. ${ }^{(3)}$ Considering some invariant properties of light harvesting, Levoy and Hanrahan reduced the all-optical function to four dimensions $(x, y, z, \theta, \varphi)$ and introduced the

*Corresponding author: e-mail: zuoyingchn@163.com

**Corresponding author: e-mail: hlguan@cnu.edu.cn

https://doi.org/10.18494/SAM.2021.3509

ISSN 0914-4935 (C) MYU K.K. https://myukk.org/ 
idea of reference planes to parametrically characterize the 4D light field by the intersection of light rays with two parallel planes. The reference planes are a parametric characterization of the 4D light field in terms of $(u, v, s, t)$, where $(u, v)$ and $(s, t)$ denote the intersections of the ray and two planes. ${ }^{(4)}$ This parametric characterization method provides the theoretical basis for a light field acquisition approach. Accordingly, the light field acquisition process is in fact the process of obtaining the intersection of light and two reference planes, and the location of the reference planes determines the type of light field acquisition equipment required. There are two main types of existing light field acquisition device: one type records light similarly to a camera array by placing a reference plane on the object side and the other type records light similarly to an alloptical camera by placing a reference plane on the image side. The camera array consists of multiple conventional cameras, ${ }^{(5,6)}$ as shown in Fig. 1, which form a virtual projection reference plane consisting of multiple lens projection centers and a virtual imaging plane consisting of multiple CMOS sensors. The camera light field is collected by acquiring the intensity of light radiation from a point in the target scene as seen from different perspectives, and the images taken by each camera can be regarded as sampled images from different angles of the light field. ${ }^{(7-9)}$ An all-optical camera consists of a main lens, a microlens array, and an imaging sensor, and the microlens array is placed before the sensor ${ }^{(10,11)}$ as shown in Fig. 2, where the camera forms two reference planes, a microlens array, and a CMOS sensor. The light field is collected by capturing the angular distribution of light at the main lens through individual microlenses.

The two types of light field acquisition device mentioned above operate mainly by the angular sampling of light. All images are recorded at a given angular resolution, treating a beam of light as a single ray. The light field is obtained by simultaneously recording the light propagation path and intensity information in one imaging session. However, regardless of whether a camera array or a plenoptic camera collects the light field, special equipment is required. Camera arrays require dozens or even hundreds of conventional cameras, resulting in the need for more equipment and making it difficult to control their time synchronization accuracy and relative position. A plenoptic camera has a simple structure compared with a camera array, and it only needs to collect the light field through one exposure; however, the

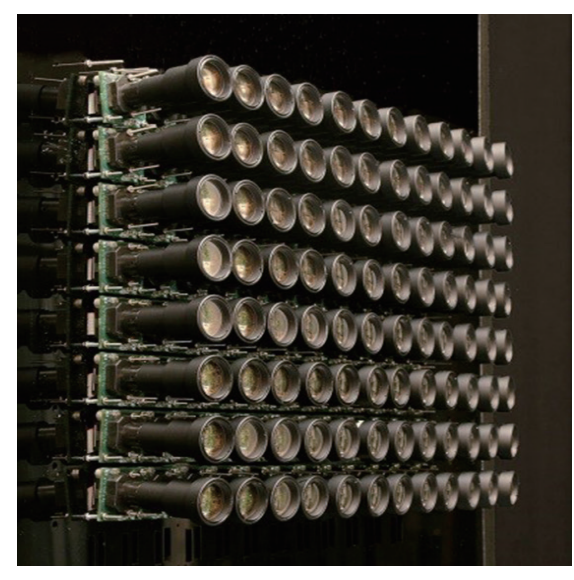

Fig. 1. (Color online) Camera array.

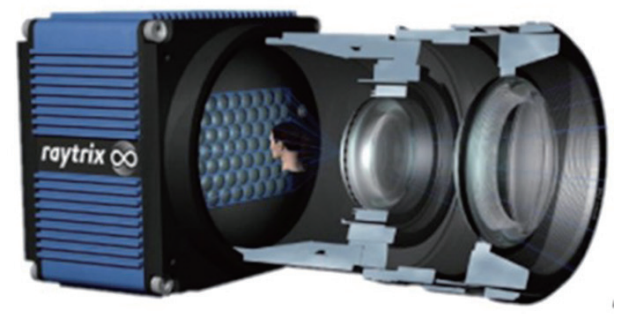

Fig. 2. (Color online) Raytrix plenoptic camera. 
angular and spatial resolutions of the light field collected with this method are both limited, which leads to a spatial resolution much lower than that of a conventional camera. Therefore, developing a simple structure without the loss of the spatial resolution for light field acquisition has become a pressing problem. If a light field is viewed as a 3D field that fills space, we can slice and sample it at different depths. The light field is acquired by recording the slice information at different depths; this sampling method is referred to as depth sampling in this paper. As shown in Fig. 3, a theoretical model of light field acquisition based on depth sampling is proposed in this paper. The main contributions are as follows:

(1) A theoretical model of light field acquisition based on depth sampling is proposed, and the theoretical transformation process from the sampled image set to the light field $L(x, y, u, v)$ is given for known slice sampled image sets $\left\{\left(x_{1}, y_{1}, d_{1}\right), \ldots,\left(x_{M}, y_{M}, d_{M}\right)\right\}$ with different depths.

(2) On the basis of the above theoretical model and with the help of the theory of image reconstruction by projection, the algorithm and technical procedure for light field acquisition from depth sampling are given.

\section{Theoretical Model of Depth Sampling Light Field Acquisition}

In the theoretical model of depth sampling light field acquisition, two mutually parallel planes are introduced to parametrically characterize the light field. As shown in Fig. 4, the main lens plane $(u, v)$ represents the reference plane in the direction of the light source and the image plane $(x, y)$ represents the reference plane of the light imaging direction. $x_{m}$ denotes different image planes and $d_{m}$ denotes different image distances.

It is assumed that depth sampling is expressed as

$$
I(x, y, d)=\left[\begin{array}{c}
x_{1}, y_{1}, d_{1} \\
\vdots \\
x_{M}, y_{M}, d_{M}
\end{array}\right],
$$
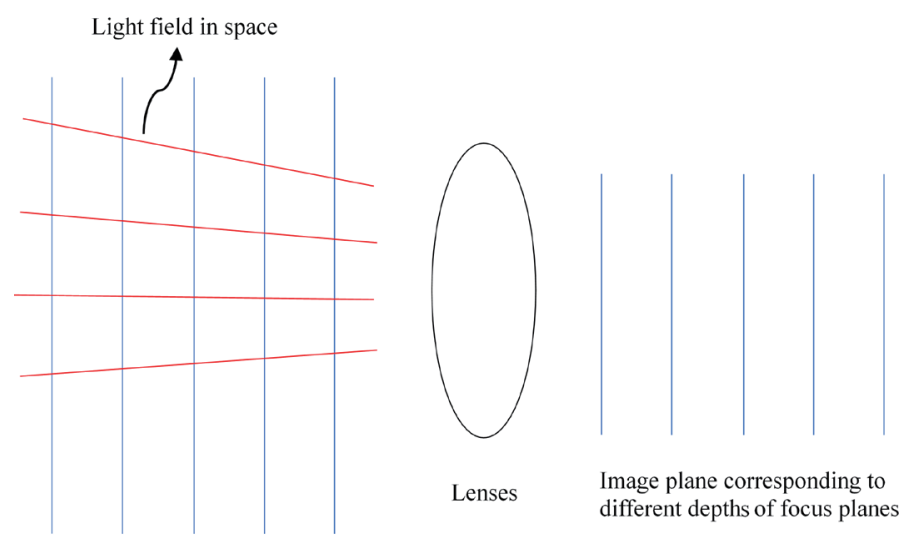

Focus planes with different depths

Fig. 3. (Color online) Theoretical model of depth sampling light field acquisition. 


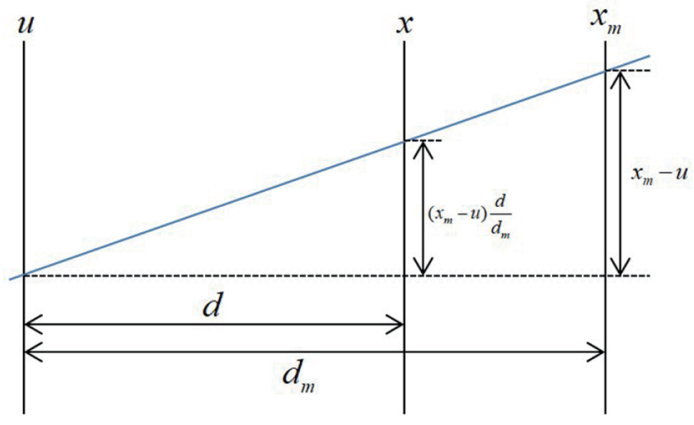

(a)

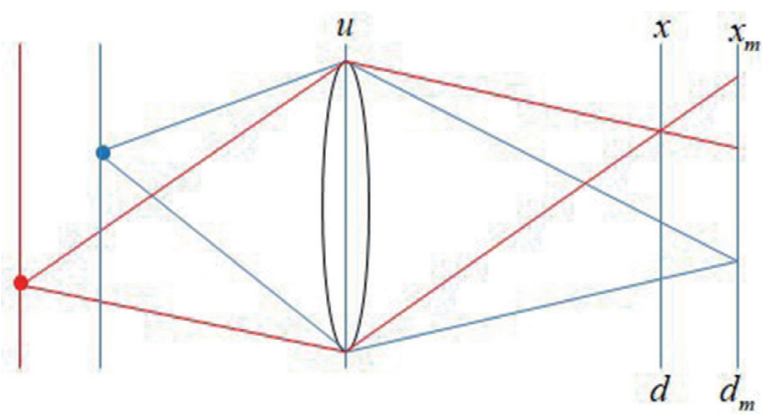

(b)

Fig. 4. (Color online) Two-plane representation model of the light field and its image in different focus planes. (a) Light field biplane representation model and (b) images made by different focus planes.

where $I(x, y, d)$ represents the pixel value at image plane $d$ and $(x, y)$.

It can be seen from Fig. 4 that for the same light, both sides of Eq. (2) are equivalent expressions:

$$
L_{d}(x, y, u, v)=L_{d_{m}}\left(x_{m}, y_{m}, u, v\right)
$$

Equation (3) can be obtained from the triangle similarity theorem:

$$
x=u+\left(x_{m}-u\right) \frac{d}{d_{m}} .
$$

In the same way, Eq. (4) can be obtained:

$$
y=v+\left(y_{m}-v\right) \frac{d}{d_{m}} .
$$

Thus, we obtain

$$
L_{d_{m}}\left(x_{m}, y_{m}, u, v\right)=L_{d}\left(u+\left(x_{m}-u\right) \frac{d}{d_{m}}, v+\left(y_{m}-v\right) \frac{d}{d_{m}}, u, v\right)
$$

Next, the depth sampling can be expressed as

$$
\begin{aligned}
I\left(x_{m}, y_{m}, d_{m}\right) & =\iint L_{d_{m}}\left(x_{m}, y_{m}, u, v\right) d u d v \\
& =\iint L_{d}\left(u+\left(x_{m}-u\right) \frac{d}{d_{m}}, v+\left(y_{m}-v\right) \frac{d}{d_{m}}, u, v\right) d u d v
\end{aligned}
$$


Transforming both sides of the equation, we obtain

$$
I\left(\frac{d}{d_{m}}\left(x_{m}-u\left(1-\frac{d_{m}}{d}\right)\right), \frac{d}{d_{m}}\left(y_{m}-v\left(1-\frac{d_{m}}{d}\right)\right), d_{m}\right)=\iint L_{d}\left(x_{m}, y_{m}, u, v\right) d u d v .
$$

where $\iint L_{d}\left(x_{m}, y_{m}, u, v\right) d u d v$ represents the projected pixel value for the same ray at the same position in each image plane.

Image reconstruction involves the projection of a 2D cross section of the object in various directions in the image plane to obtain a series of $1 \mathrm{D}$ projection functions. Then, the $2 \mathrm{D}$ cross section of the object is reconstructed using these $1 \mathrm{D}$ projection functions. ${ }^{(12)}$ The most commonly used image reconstruction method is the filtered backprojection algorithm. In this paper, for light field multiview image reconstruction, the accumulation method in the filtered backprojection algorithm is also used. The filtered backprojection algorithm is a spatial processing method based on the Fourier transform. This algorithm performs convolution processing on the projections from each acquisition projection angle before backprojection to reduce the shape artifacts caused by the point spread function, thereby improving the quality of the reconstructed image. ${ }^{(13)}$ According to the theory of projection reconstructed images, the image of any point can be regarded as the integral of all the light rays passing through the point at different angles. The algorithm is expressed as

$$
i=\sum_{T} P_{\theta}
$$

where $i$ is the pixel value of the point, $P_{\theta}$ is the projection value of the ray passing through the point at a certain angle $\theta$, and $T$ is the number of projection angles.

Each image in depth sampling can also be seen as a 2D projection of a 4D light field. The projection on each image plane is $\iint L_{d}\left(x_{m}, y_{m}, u, v\right) d u d v$. The number of depth samples is equivalent to that projection angles $T$. Then, the $4 \mathrm{D}$ light field recovered from the depth sampling using Eq. (8) can be expressed as

$$
\begin{aligned}
L_{r e c}(x, y, u, v)= & \iint L_{d}\left(x_{1}, y_{1}, u, v\right) d u d v+\cdots+\iint L_{d}\left(x_{m}, y_{m}, u, v\right) d u d v \\
= & I\left(\frac{d}{d_{1}}\left(x_{1}-u\left(1-\frac{d_{1}}{d}\right)\right), \frac{d}{d_{1}}\left(y_{1}-v\left(1-\frac{d_{1}}{d}\right)\right), d_{1}\right)+\cdots \\
& I\left(\frac{d}{d_{m}}\left(x_{m}-u\left(1-\frac{d_{m}}{d}\right)\right), \frac{d}{d_{m}}\left(y_{m}-v\left(1-\frac{d_{m}}{d}\right)\right), d_{m}\right) \\
= & \sum_{m=1}^{M} I\left(\frac{d}{d_{m}}\left(x_{m}-u\left(1-\frac{d_{m}}{d}\right)\right), \frac{d}{d_{m}}\left(y_{m}-v\left(1-\frac{d_{m}}{d}\right)\right), d_{m}\right) \\
= & \sum_{m=1}^{M} I\left(\frac{1}{\alpha_{m}}\left(x_{m}-u\left(1-\alpha_{m}\right)\right), \frac{1}{\alpha_{m}}\left(y_{m}-v\left(1-\alpha_{m}\right)\right), d_{m}\right) .
\end{aligned}
$$


In this equation, $\alpha_{m}=d_{m} / d$, which represents the image distance ratio, and $L_{r e c}(x, y, u, v)$ is the collected 4D light field. For a given $(u, v)$, the transmission direction of light can be determined, which is equivalent to the determination of a virtual camera. Taking images of light in the direction of $(x, y)$ given different $(u, v)$ values, we can obtain images having different perspectives. $M$ represents the number of depth samples, and d represents the reference image plane, which can be of any $d_{m}$.

\section{Results and Discussion}

\subsection{Light field acquisition results based on depth sampling}

Depth sampling can be understood as a set of images $I(x, y, d)$ focused on different depths of the target scene, representing a sliced sampling of different depths of the light field. This sampling approach is different from that of common devices or methods that use camera or microlens arrays to sample light field angles. If depth samples are regarded as images with different focusing distances, then depth sampling can be achieved with simpler equipment, such as ordinary optical cameras. The focal length of the camera is fixed, and the sampled data of different depth slices are obtained by acquiring images with different depths of focus. We use a Canon 5D Mark III camera as the experimental device. In the experiment, the device position is fixed to obtain slice samples having different depths in a target scene.

The target scene for this article is composed of four playing cards, with each card representing a focus plane. The reason for choosing playing cards is that they are easy to focus on, and the movement of the viewing angle can be clearly seen after collecting the light field. The depth sampling data obtained with the Canon camera are formulated as $\left\{\left(x_{1}, y_{1}, d_{1}\right), \ldots,\left(x_{4}, y_{4}, d_{4}\right)\right\}$. Four images with different focus planes completely cover the entire experimental scene. In addition, the $(x, y)$ resolution is $1920 \times 1280$. To achieve better results, the camera control software digiCamControl is used to control the camera from a computer. The depths of focus of the four images are $0.75,0.84,0.96$, and $1.03 \mathrm{~m}$. To minimize the impact of the depth of the field on data collection, the focal length of the equipment used in the experiment is adjusted to $105 \mathrm{~mm}$, and the aperture is adjusted to 4.0. When the depth of focus is $1 \mathrm{~m}$, the depth of field is approximately $10 \mathrm{~cm}$, and these depths of focus obtain more ideal images. The images acquired with different depths of focus are shown in Fig. 5.

In this study, Eq. (9) represents the light field collected from the depth sampling. In addition, subaperture images are used to visualize light fields. According to Eq. (9), given different $(u, v)$ values, images $(x, y)$ with different perspectives are obtained, where $u$ represents the viewing angle in the horizontal direction and $v$ represents the viewing angle in the vertical direction. We set the values of $(u, v)$ to $(20,0),(0,0)$ and $(-20,0)$, where $(0,0)$ represents the central viewing angle. The value in the vertical direction $v$ remains fixed at 0 , and that in the horizontal direction $u$ is set to different values to observe the movement of the viewing angle. The obtained images $(x, y)$, which have different perspectives, are shown in Fig. 6. The upper half of each image shows the acquired subaperture image, and the lower half is a partially enlarged view from the left of the subaperture image. 


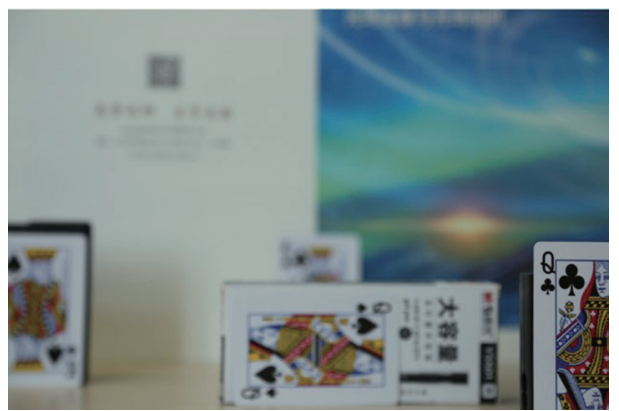

(a)

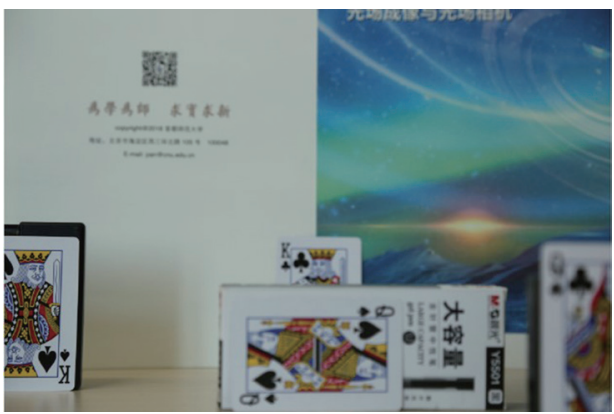

(c)

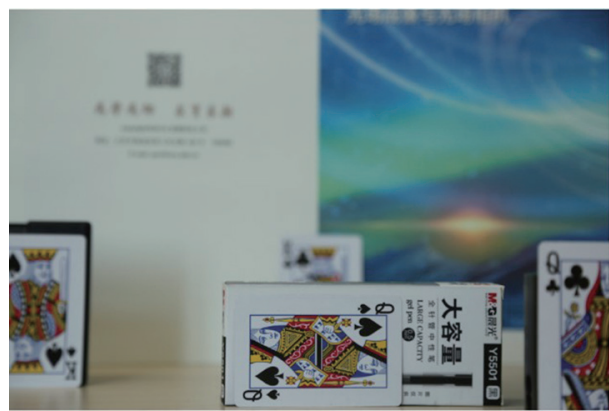

(b)

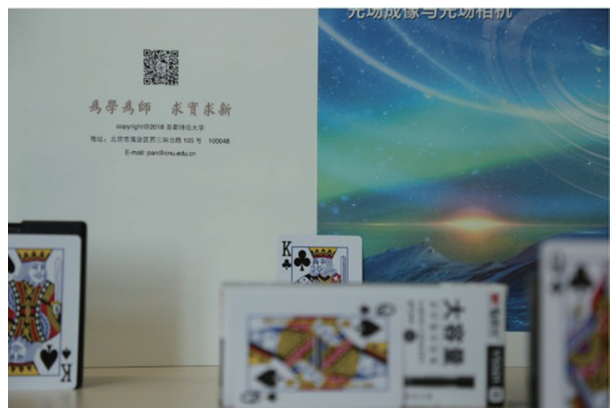

(d)

Fig. 5. (Color online) Images with different depths of focus captured by Canon camera. Depths of focus: (a) 0.75 , (b) 0.84 , (c) 0.96 , and (d) $1.03 \mathrm{~m}$.

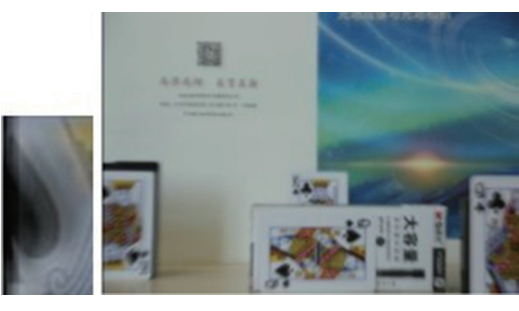

(a)

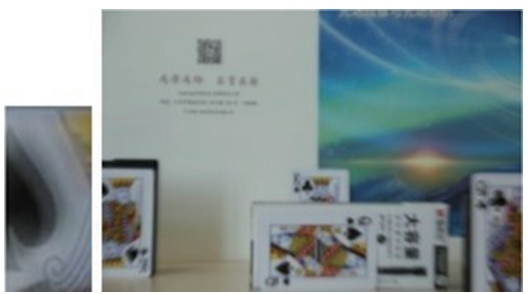

(b)

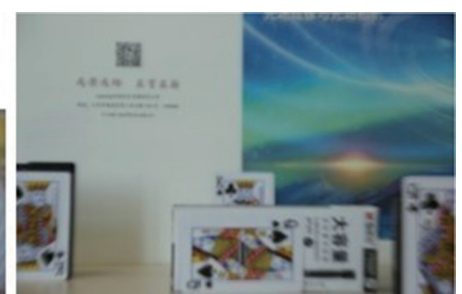

(c)

Fig. 6. (Color online) Light field acquisition results obtained by depth sampling. (a) $(u, v)=(20,0)$, (b) $(u, v)=(0,0)$, and $(\mathrm{c})(u, v)=(-20,0)$.

The experimental results also demonstrate that given different $(u, v)$ values, subaperture images $(x, y)$ of different viewing angles can be obtained. In the experimental scene above, each playing card is selected as a focus plane, and the four cards completely cover the entire experimental scene. To better characterize the experiment and discuss the effect of the number of depth samples at the same time, we choose two and three depth samples that do not completely cover the experimental scene for comparison. The experimental results are shown in Fig. 7.

Since there is no true image for reference in this study, the Tenengrad, Laplacian, and variance functions are selected to evaluate the sharpness of the three images in Fig. 7. The Tenengrad and Laplacian functions are gradient-based functions that can be used to detect 


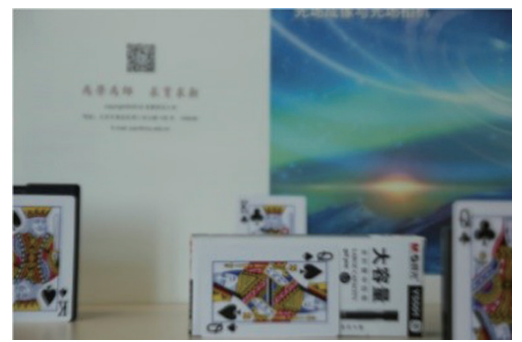

(a)

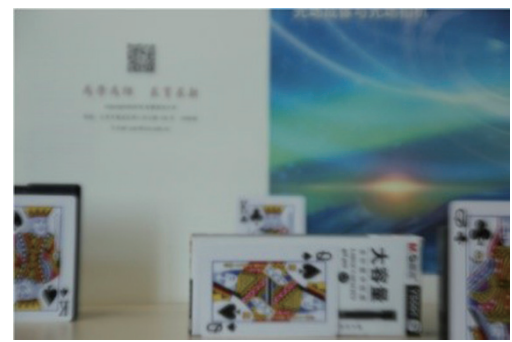

(b)

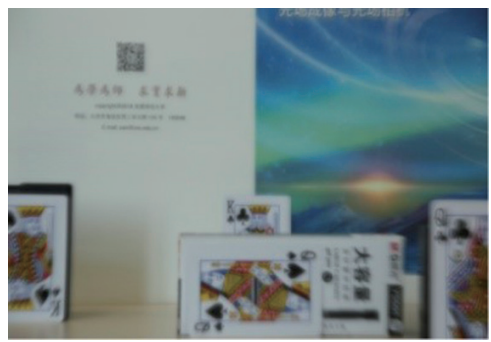

(c)

Fig. 7. (Color online) Light field acquisition results from different numbers of depth samples. Numbers of depth samples are (a) 2, (b) 3, and (c) 4 .

Table 1

Image clarity evaluation results for different numbers of depth samples.

\begin{tabular}{lccc}
\hline Number of depth samples & 2 & 3 & 4 \\
\hline Tenengrad function & 0.5110 & 0.6120 & 0.6910 \\
\hline Laplacian function & 0.8562 & 0.8876 & 0.9621 \\
\hline Variance function & 1663.63 & 1710.35 & 1796.29 \\
\hline
\end{tabular}

whether an image is sharp and has sharp edges. The clearer the image is, the larger the resulting value. ${ }^{(14,15)}$ The variance function is a measure of the degree of dispersion between discrete data and the expectation of probability theory. Since a clear image has a larger grayscale difference between pixels than a blurred image, the variance function can be used to evaluate the sharpness of the image; the clearer the image, the larger the variance. The three sharpness evaluation functions are used to quantitatively evaluate the three subaperture images generated above, and the results are shown in Table 1.

It can be seen from these results that the greater the number of depth samples, the clearer the collected light field image. When the depth samples cover the entire experimental scene, the collected light field image is clearer than an image that does not completely cover the entire experimental scene. This is mainly because when the depth samples do not cover the entire experimental scene, part of the experimental scene cannot be clearly focused, which causes the image to become blurred. The light field subaperture image collected using Eq. (9) is not as clear as an image that completely covers the experimental scene.

\subsection{Comparison of light field acquisition results obtained by depth and angular sampling methods}

The depth-sampling-based light field image acquisition method only requires the use of ordinary cameras to acquire images in different focus planes to achieve light field computational imaging, which is very different from the angular-sampling-based light field acquisition method in terms of the acquisition model and equipment used. However, the depth-sampling-based method requires multiple consecutive shots of the target scene, which is more conducive to light field image acquisition in static or slow-moving experimental scenes. This is obviously different 
from the light field image acquisition method that uses a plenoptic camera to collect the light field in one shot. To better verify the experimental results of our proposed method, the popular Lytro Illum V2 plenoptic camera is used to collect light field data from the same experimental scene, and the light field images acquired by the two cameras are compared in terms of their sharpness and acquisition effects.

The Lytro Illum V2 plenoptic camera produces approximately 40 million effective pixels, the capture sensor has a resolution of $7728 \times 5368$, the number of microlens arrays is $541 \times 434$, the angular resolution is $15 \times 15$, and the number of pixels behind each microlens is 225 . For this article, two cameras, Lytro Illum V2 and Canon 5D Mark III, are used to perform angular and depth sampling methods for the same experimental scene. To reduce the effects of other camera parameters on the experimental results, the focal lengths of both cameras are set to $105 \mathrm{~mm}$. The sampling results of the two methods are shown in Fig. 8.

The light field image collected by the proposed method is a subaperture image. Therefore, it is necessary to decode the original light field image captured by the Lytro Illum V2 camera into a subaperture image to compare the collection effects. The angular resolution of the camera is $15 \times 15$, which means that a total of $15 \times 15$ subaperture images can be decoded. The $(u, v)$ values given when collecting light field images by depth sampling are $(20,0),(0,0)$ and $(-20,0) .(0,0)$ represents the central viewing angle, and the value of $\mathrm{v}$ in the vertical direction is fixed. For consistency, we fix the subaperture image of the camera's central viewing angle and its vertical parallax, and only the leftmost and rightmost subaperture images of the horizontal parallax are

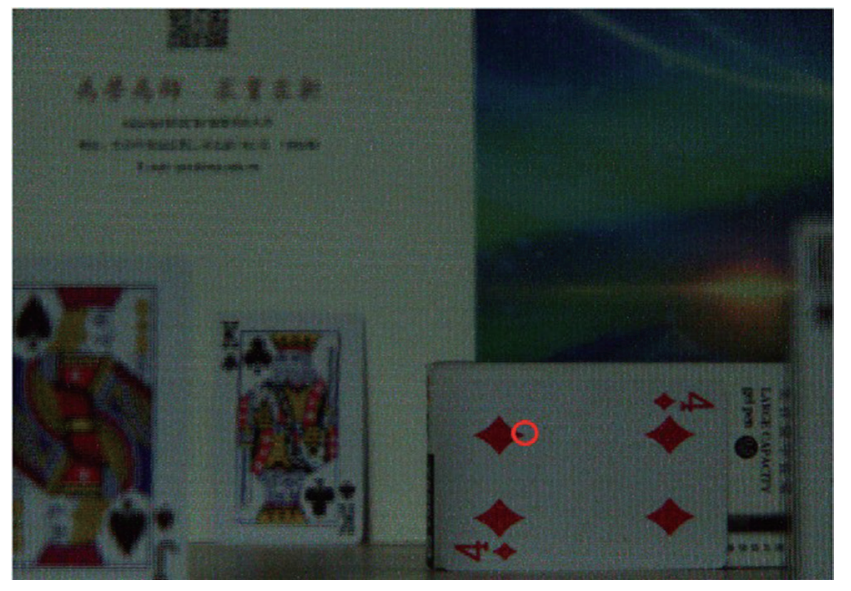

(a)
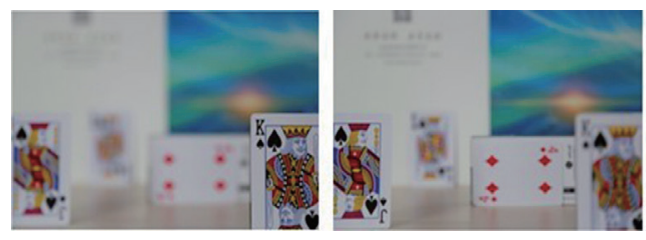

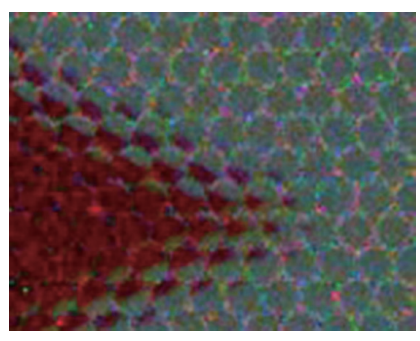

(b)
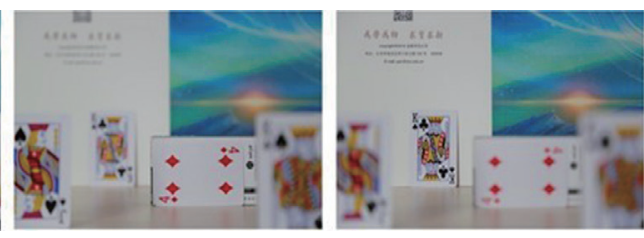

(c)

Fig. 8. (Color online) Results of sampling with two methods. (a) Raw image of light field taken by Lytro camera, (b) enlargement of image in red circle, and (c) depth-sampled image obtained by Canon camera. 
used for effect comparison. The subaperture images of the light field collected by the two methods are shown in Fig. 9. The small image on the left of each image is a partially enlarged view to clearly show the movement of the subaperture image viewing angle.

For the light field subaperture images collected by the two methods, the angular resolution of the light field image collected by depth sampling can reach that of the light field image collected by angular sampling. The spatial resolution of the light field image collected by depth sampling is $1920 \times 1280$, which is the same as the size of the original sensor. The spatial resolution of the light field image collected by angular sampling is $625 \times 433$, which is much smaller than the size of the original sensor of $7728 \times 5328$.

To better verify the effects of the proposed method from a quantitative perspective, the three sharpness evaluation functions mentioned above are again used to evaluate the sharpness of the light field images collected by angular and depth sampling methods. The results are shown in Table 2.

It can be seen from the table that the light field subaperture image collected by depth sampling is not as clear as that collected by angular sampling. However, the difference between the two sampling methods is very small. The reason for the less clear image is that the light field depth sampling algorithm used for the proposed method directly adds the data in the depth samples, which causes the acquired subaperture image to become blurred.
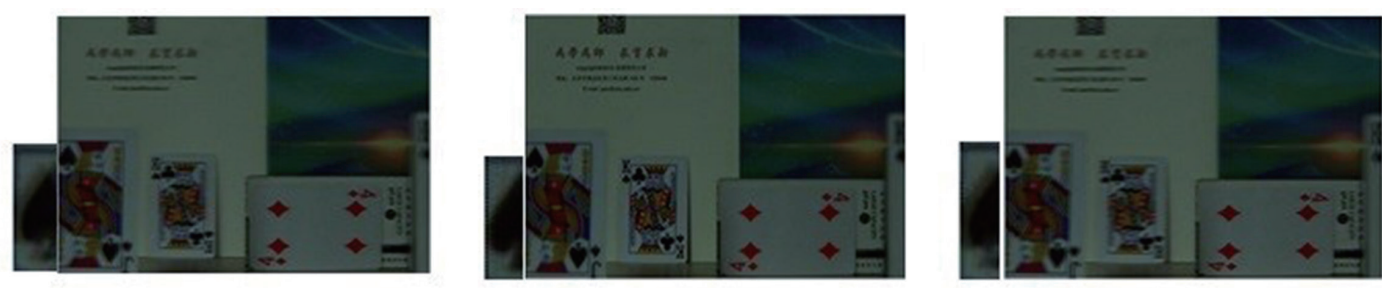

Angular Sampling
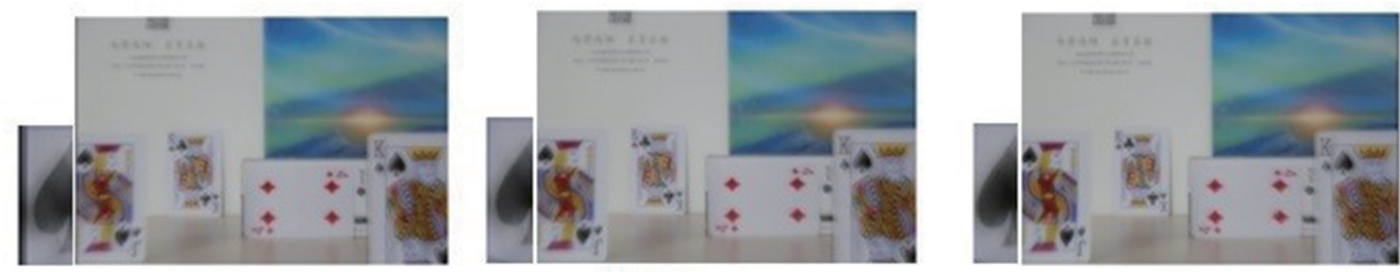

Depth Sampling

Fig. 9. (Color online) Light field collection results of different sampling methods.

Table 2

Image clarity evaluation results of different sampling methods.

\begin{tabular}{lcc}
\hline Sampling method & Angular sampling & Depth sampling \\
\hline Tenengrad function & 0.6120 & 0.5110 \\
\hline Laplacian function & 0.8876 & 0.8562 \\
\hline Variance function & 1710.35 & 1663.63 \\
\hline
\end{tabular}


From the experiment in Sect. 3.1, we find that by setting different $(u, v)$ values in the depth sampling algorithm, we can obtain subaperture images $(x, y)$ with different viewing angles. To explore the effects of different $u$ values on the collected subaperture images, we employ four different $u$ values in our experiments; the experimental results are shown in Fig. 10.

The three sharpness evaluation functions are also used to evaluate the sharpness of the light field images collected after setting different $u$ values. The results are shown in Table 3 .

Figure 10 and Table 3 show that the larger the $u$ value, the more blurred the acquired light field subaperture image. Equation (9) shows that as $u$ increases, the amplitude of the image movement becomes larger, which makes the image more blurred when the image movement is added. A similar effect is seen in the subaperture image collected by the Lytro Illum V2 camera. The subaperture image quality is highest at the center of the microlens and deteriorates with increasing distance from the center.

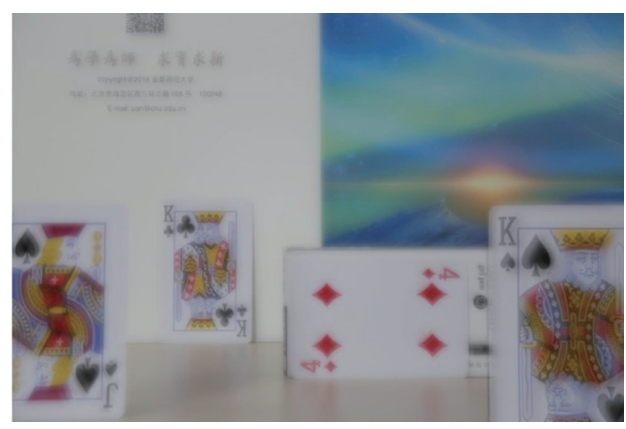

(a)

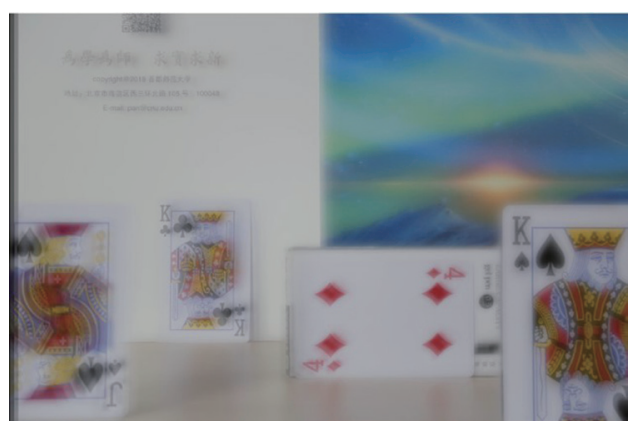

(c)

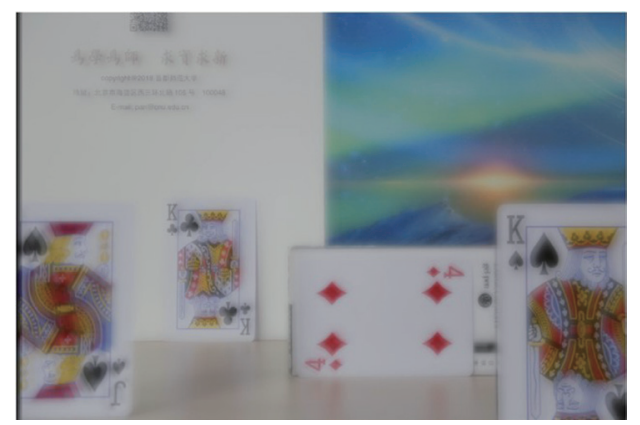

(b)

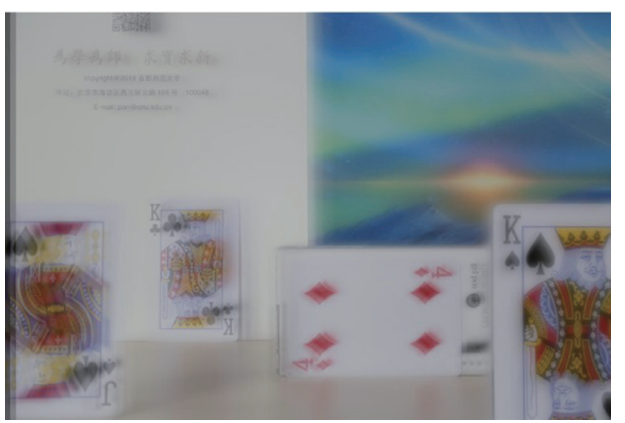

(d)

Fig. 10. (Color online) Light field collection results for different $u$ values. (a) $(u, v)=(0,0)$, (b) $(u, v)=(40,0)$, (c) $(u, v)=(80,0)$, and $(\mathrm{d})(u, v)=(120,0)$.

Table 3

Evaluation results of image sharpness with different $u$ values.

\begin{tabular}{lcccc}
\hline$(u, v)$ & $(0,0)$ & $(40,0)$ & $(80,0)$ & $(120,0)$ \\
\hline Tenengrad function & 0.4431 & 0.4291 & 0.3922 & 0.3532 \\
\hline Laplacian function & 0.7381 & 0.6488 & 0.6012 & 0.5542 \\
\hline Variance function & 1169.50 & 1022.61 & 956.92 & 932.12 \\
\hline
\end{tabular}




\section{Conclusions}

We propose a new light field acquisition method that enables the depth sampling of the target scene, acquiring different depth images of the target scene, and then recovers the 4D light field from the depth sampling data. The clarity of the light field subaperture images collected by this method and by the traditional angular sampling method is evaluated. The experimental results show that the light field images collected by the depth sampling method proposed in this paper do not require special hardware, and the spatial resolution can reach the sensor size. The spatial resolution of the light field image collected by the traditional angular sampling method is much smaller than the size of the sensor. The clarity of a light field image collected by the proposed method is very close to that of the light field image collected by the angular sampling method. This method provides a more concise approach to the acquisition of light field images in computational imaging technology.

\section{Acknowledgments}

This work was supported by the National Key R\&D Program of China (No. 2018YFC1800904); Capacity Building for Sci-Tech Innovation - Fundamental Scientific Research Funds (No. 20530290078)

\section{References}

1 M. Levoy: Computer 39 (2006) 46. https://doi.org/10.1109/MC.2006.270

2 G. Wetzstein, I. Ihrke, D. Lanman, and W. Heidrich: Comput. Graphics Forum 30 (2011) 2397. https://doi. org/10.1111/j.1467-8659.2011.02073.x

3 E. H. Adelson and J. R. Bergen: Comput. Models Visual Process. 1 (1991) 3.

4 M. Levoy and P. Hanrahan: Proc. 23rd Annu. Conf. Computer Graphics and Interactive Techniques 12 (1996) 31. https://doi.org/10.1145/237170.237199

5 B. Wilburn, N. Joshi, V. Vaish, EV. Talvala, E. Antunez, A. Barth, A. Adams, M. Horowitz, and M.Levoy: ACM SIGGRAPH 2005 Papers (2005) 765. https://doi.org/10.1145/1186822.1073259

6 V. Vaish, B. Wilburn, N. Joshi, and M. Levoy: IEEE Comput. Soc. 1 (2004) 1. https://doi.org/10.1109/ CVPR.2004.1315006

7 V. Vaish, G. Garg, E. Talvala, E. Antunez, B. Wilburn, M. Horowitz, and M. Levoy: 2005 IEEE Computer Society Conf. Computer Vision and Patter Recognition - Workshops (2005) 129. https://oi.org/10.1109/ CVPR.2005.537

8 N. Joshi, S. Avidan, W. Matusik, and D. J. Kriegman: 2007 IEEE 11th Int. Conf. Computer Vision (2007) 1. https://doi.org/10.1109/ICCV.2007.4409032

9 M. Cho and B. Javidi: Opt. Lett. 33 (2008) 2737. https://doi.org/10.1364/0L.33.002737

10 E. H. Adelson and J. Y. A. Wang: IEEE Trans. Pattern Anal. Mach. Intell.14 (1992) 99.

11 R. Ng, M. Levoy, M. Brédif, G. Duval, M. Horowitz, and P. Hanrahan: Doctoral Dissertation, Light Field Photography with a Hand-held Plenoptic Camera (Stanford University, 2005). https://hal.archives-ouvertes.fr/ hal-02551481

12 R. M. Lewitt: Proc. IEEE 71 (1983) 390. https://doi.org/10.1109/PROC.1983.12597

13 S. Pan and A. Kak: IEEE Trans. Acoust. Speech Signal Process. 31 (1983) 1262. https://doi.org/10.1109/ TASSP.1983.1164196

14 Y. Yao, B. Abidi, N. Doggaz, and M. Abidi: Visual Inf. Process. XV 6246 (2006) 62460G. https://doi. org/10.1117/12.664751

15 R. Redondo, G. Cristóbal, G. B. Garcia, O. Deniz, J. Salido, M. del Milagro Fernandez, J. Vidal, J. C. Valdiviezo, R. Nava, B. Escalante-Ramirez, and M. Garcia-Rojo: J. Biomed. Opt. 17 (2012) 036008. https://doi. org/10.1117/1.JBO.17.3.036008 


\section{About the Authors}

Fuzhou Duan received his B.S. degree from Wuhan University, China, in 2001 and his Ph.D. degree from Capital Normal University, China, in 2007. Since 2007, he has been an associate professor at Capital Normal University. His research interests are in aerial remote sensing data collection technology and its application in the fields of resources, environment, natural disasters, and so forth. (5482@cnu.edu.cn)

Ying Zuo received her B.S. degree from Chuzhou University, China, in 2019. Since 2019, she has been studying for an M.S. degree at Capital Normal University. Her research interests are in light field imaging technology and its application. (zuoyingchn@163.com)

Hongliang Guan received his B.S. degree from Wuhan University, China, in 1992 and his M.S. and Ph.D. degrees from The University of Tokyo, Japan, and Capital Normal University, China, in 2001 and 2010, respectively. Since 2016, he has been a professor at Capital Normal University. His research interests are in light field imaging technology and its application.

(hlguan@cnu.edu.cn)

Tian Guo received his B.S. degree from Jiangxi University of Science and Technology, China, in 2017 and his M.S. degree from Capital Normal University, China, in 2020. Since 2020, he has worked as an engineer in Wuhan University Gonow Information Technology Co., Ltd. His research interests are in light field imaging technology and its application.

(2503840754@qq.com) 